\title{
A Study of Nonholonomic Deformations of Nonlocal Integrable Systems Belonging to the Nonlinear Schrödinger Family
}

\author{
I. Mukherjee, P. Guha
}

The nonholonomic deformations of nonlocal integrable systems belonging to the nonlinear Schrödinger family are studied using the bi-Hamiltonian formalism as well as the Lax pair method. The nonlocal equations are first obtained by symmetry reductions of the variables in the corresponding local systems. The bi-Hamiltonian structures of these equations are explicitly derived. The bi-Hamiltonian structures are used to obtain the nonholonomic deformation following the Kupershmidt ansatz. Further, the same deformation is studied using the Lax pair approach and several properties of the deformation are discussed. The process is carried out for coupled nonlocal nonlinear Schrödinger and derivative nonlinear Schrödinger (Kaup Newell) equations. In the case of the former, an exact equivalence between the deformations obtained through the bi-Hamiltonian and Lax pair formalisms is indicated.

Keywords: nonlocal integrable systems, nonlinear Schrödinger equation, Kaup-Newell equation, bi-Hamiltonian system, Lax method, nonholonomic deformation

Received May 01, 2019

Accepted September 03, 2019

Indranil Mukherjee

indranil.m11@gmail.com

School of Natural and Applied Sciences,

Maulana Abul Kalam Azad University of Technology,

BF 142, BF Block, Sector 1, Bidhannagar, Kolkata, West Bengal, 700064 India

Partha Guha

partha@bose.res.in

S. N. Bose National Centre for Basic Sciences,

JD Block, Sector III, Salt Lake, Kolkata, 700098 India 


\section{Introduction}

The importance of completely integrable systems in different areas such as water waves, plasma physics, field theory, nonlinear optics, lattice dynamics etc. can hardly be overemphasized [1]. The standard technique to study integrable models is by using the Lax pair, using the zero curvature or flatness condition [2]. Systems are considered to be integrable when they contain infinitely many conserved quantities which lead to the stability of the soliton solutions. These constants of motion determine the system dynamics, thereby allowing solutions by the method of inverse scattering transform (IST) in appropriate variables [3, 4]. The integrable systems are found to possess a local bi-Hamiltonian structure $[5,6]$. It is well known that, starting from a suitably chosen spectral problem, one can set up a hierarchy of nonlinear evolution equations. This is attained by using recurrence relations which enable construction of the hierarchy through use of certain polynomial functions, generating the Lax pairs and thereby the different equations of the hierarchy. One of the ongoing challenges in the study of integrable systems is to construct such systems associated with nonlinear evolution equations of physical significance.

Two relatively recent developments in the field of integrable systems seem to have an important impact on the growth and orientation of the field. The first one was the initiation of the concept of nonholonomic deformation of integrable models in 2008, while the second event was the introduction of nonlocal integrable systems in 2013. Considering the latter, we note that the nonlinear Schrödinger (NLS) equation, in one space and one time $(1+1)$ variables, is an extremely well-known classical nonlinear integrable equation appearing in diverse physical systems $[7,8]$ encompassing nonlinear optics, plasma physics, fluid mechanics and also mathematical fields like differential geometry [9]. A new integrable reduction of this well-known equation, called the nonlocal nonlinear Schrödinger equation, was obtained in [10]. This equation is PT symmetric [28-30], i.e., it is symmetric under the parity-time transform $x \rightarrow-x$, $t \rightarrow-t, q \rightarrow q^{*}$. It has been introduced as a mathematical model to discuss wave propagation in PT symmetric nonlinear media [11-13]. The idea originated in some past work carried out by Fokas and others in relation to integrable boundary-value problems for the nonlinear Schrödinger equation [14-18] as well as on the nonlocal formulation of water waves [19]. A multidimensional analog of the nonlocal nonlinear Schrödinger equation was also discovered in [20]. A variety of integrable nonlocal nonlinear equations were obtained through symmetry reductions of the AKNS scattering problem, the nonlocality appearing in both space and time or time alone [21]. Examples include, apart from nonlocal NLS, the modified KdV, sine-Gordon, $(1+1)-$ and $(2+1)$-dimensional three-wave interaction, derivative NLS, Davey-Stewartson equations etc. Several related studies were undertaken subsequently [22-27] which dealt with different aspects of nonlocal integrable systems.

The second development, viz., the nonholonomic deformation of integrable systems is one in which the system is perturbed in such a manner that, under suitable differential constraints on the perturbing function, the system retains its integrability. It was shown by Karasu-Kalkani et al. [31] that the integrable 6th-order KdV equation represented a nonholonomic deformation(NHD) of the celebrated KdV equation. The terminology "nonholonomic deformation" was used by Kupershmidt [32]. In [33] a matrix Lax pair, the N-soliton solution using inverse scattering transform and a two-fold integrable hierarchy were obtained for the nonholonomic deformation of the $\mathrm{KdV}$ equation. In [34] the work was extended to include the nonholonomic deformation of both $\mathrm{KdV}$ and $\mathrm{mKdV}$ equations along with their symmetries, hierarchies and integrability. The nonholonomic deformation of derivative NLS and Lenells - Fokas equations was discussed in [35]. In this paper, deformed integrable versions of these equations were obtained 
with some arbitrary functions of $t$ as coefficients and solutions of these equations were found to give rise to the phenomenon of accelerating solitons as solutions of these deformed equations with a suitable choice of the time-dependent coefficients. The deformation of generalized $\mathrm{KdV}$ type equations was taken up in [36], where emphasis was put on the geometrical aspect of the problem. Kupershmidt's infinite-dimensional construction was extended in [37] to obtain nonholonomic deformation of a wide class of coupled KdV systems, all of which are generated from the Euler - Poincaré - Suslov flows. A comparative study encompassing two different types of deformations, viz., nonholonomic and quasi-integrable, of equations in the NLS and DNLS hierarchy was undertaken in [38].

\subsection{The purpose, result and structure of the paper}

Consider a coupled system of evolution equations given by

$$
i q_{t}=q_{x x}-2 q^{2} r, \quad-i r_{t}=r_{x x}-2 r^{2} q,
$$

where $q(x, t)$ and $r(x, t)$ are potentials of the well-known AKNS $2 \times 2$ linear scattering problem. We obtain the celebrated NLS equation when $r=\sigma q^{*}, \sigma=\mp 1$. As discussed above, Ablowitz and Musslimani [10] obtained a new interesting reduction of the AKNS scattering problem, given by $r(x, t)=\sigma q^{*}(-x, t)$. This leads to the integrable nonlocal NLS equation having profound applications in PT symmetric quantum physics and optics, giving rise to the name PT symmetric NLS equation (or PTNLS in short). Ablowitz and Musslimani [10] developed the inverse scattering transform for decaying data and obtained a breathing soliton solution.

More recently, Ablowitz and Musslimani [21] found two more reductions of the AKNS scattering problem leading to interesting nonlocal NLS type equations. These are given by $r(x, t)=\sigma q(-x,-t)$ and $r(x, t)=\sigma q(x,-t)$, and the equations which are obtained from these two reductions are called reverse space-time NLS (RSTNLS) and reverse time NLS (RTNLS) equations, respectively. Ablowitz and Musslimani have found many other nonlocal integrable equations such as nonlocal modified Korteweg-de Vries equation, nonlocal Davey - Stewartson equation, nonlocal sine-Gordon equation, and nonlocal (2+1)-dimensional three-wave interaction equations. The work carried out by Ablowitz and Musslimani has led to a flurry of activities on nonlocal reductions of systems of integrable equations.

The purpose of the present work is to tie together the two relatively recent developments in the field of integrable systems by examining the effect of nonholonomic deformation on nonlocal integrable systems. The nonlocal systems are obtained by imposing suitable symmetry reductions on one of the dynamical variables occurring in the Lax pair. On the other hand, the deformation could be achieved either starting from the bi-Hamiltonian structure of the system of equations following the method adopted in [32] or by inserting perturbing functions in the temporal component of the Lax pair. The study is undertaken in respect of the NLS and the DNLS equations by first obtaining the corresponding nonlocal systems and then subjecting them to nonholonomic deformations. The latter is implemented using both approaches mentioned above. The NLS equation is chosen as a test platform in view of its generic nature and a wide range of applications.

The results obtained indicate the following:

(i) nonlocality gets introduced not only in the dynamical variables, but also in the perturbing variables;

(ii) the perturbing variables enter as "source" terms and make the deformed equations inhomogenous compared to the original integrable systems;

RUSSIAN JOURNAL OF NONLINEAR DYNAMICS, 2019, 15(3), 293-307 
(iii) the bi-Hamiltonian approach shows that the constraints on the deforming functions are integro-differential in nature;

(iv) an exact equivalence is easy to establish between the bi-Hamiltonian and Lax pair approaches in the case of the nonlinear Schrödinger (NLS) equation. The results become more complex in the case of the Kaup-Newell system of equations.

The nonholonomic deformation of nonlocal nonlinear Schrödinger equation leads to Eq. (36), while the use of the derivative nonlinear Schrödinger equation (Kaup-Newell system) gives rise to the system of equations (71) and (72), the latter being nonautonomous in nature, containing time-dependent arbitrary coefficients, and may be regarded as some kind of generalization of the Lennels - Fokas equations. The solutions of these systems may be worked out using the methods of inverse scattering transform $[3,4,23]$ as well as the methods discussed in [39, 40]. However, such solutions have not been attempted in this work since our primary focus is on constructing the nonholonomic deformed system of nonlocal equations using the two different techniques mentioned above.

The rest of the paper is organized as follows: Section 2 discusses the nonlocal nonlinear Schrödinger (NNS)equation and its bi-Hamiltonian structure. Section 3 demonstrates the nonholonomic deformation of the NNS system by using the Lax pair approach as well as the Kupershmidt prescription and establishes the equivalence of the two approaches. Section 4 considers the derivative NLS using the Kaup - Newell equation and derives the nonlocal counterpart. Section 5 is devoted to the study of nonholonomic deformation of the nonlocal Kaup-Newell system. Section 6 lists possible outcomes and indicates how the study may be carried forward.

\section{The nonlocal nonlinear Schrödinger equation and its bi-Hamiltonian structure}

We start with the Lax pair of the coupled nonlinear Schrödinger equations given by

$$
\begin{aligned}
U & =-i \lambda \sigma_{3}+q(x, t) \sigma_{+}+r(x, t) \sigma_{-}, \\
V & =\left(2 i \lambda^{2}+i q(x, t) r(x, t)\right) \sigma_{3}-2 \lambda\left(q(x, t) \sigma_{+}\right. \\
& \left.+r(x, t) \sigma_{-}\right)+i\left(-q_{x}(x, t) \sigma_{+}+r_{x}(x, t) \sigma_{-}\right) .
\end{aligned}
$$

The coupled NLS equations in terms of both $q$ and $r$ are given by

$$
q_{t}=-i q_{x x}+2 i q^{2} r, \quad r_{t}=i r_{x x}-2 i r^{2} q,
$$

and can be obtained as consistency equations by imposing the usual zero-curvature condition:

$$
U_{t}-V_{x}+[U, V]=0 .
$$

The bi-Hamiltonian structures of the pair of NLS equations are given by

$$
\begin{aligned}
B^{1} & =\left(\begin{array}{cc}
0 & -i \\
i & 0
\end{array}\right), \\
B^{2} & =\left(\begin{array}{cc}
q \partial_{x}^{-1} q & \frac{1}{2} \partial_{x}-q \partial_{x}^{-1} r \\
\frac{1}{2} \partial_{x}-r \partial_{x}^{-1} q & r \partial_{x}^{-1} r
\end{array}\right),
\end{aligned}
$$


and the corresponding conserved densities are

$$
H^{1}=-\int\left(q_{x} r_{x}+q^{2} r^{2}\right) d x \quad \text { and } \quad H^{2}=i \int\left(r_{x} q-q_{x} r\right) d x
$$

Under the standard AKNS symmetry, we put

$$
r(x, t)=\sigma q^{*}(x, t)
$$

On putting $\sigma=1$, Eq. (3) reduces to

$$
q_{t}(x, t)=-i q_{x x}(x, t)+2 i q^{2}(x, t) q^{*}(x, t), \quad q^{*}(x, t)_{t}=i q_{x x}^{*}(x, t)-2 i q^{* 2}(x, t) q(x, t) .
$$

Under this symmetry, the Hamiltonian structures are

$$
\begin{aligned}
B^{1} & =\left(\begin{array}{cc}
0 & -i \\
i & 0
\end{array}\right), \\
B^{2} & =\left(\begin{array}{cc}
q \partial_{x}^{-1} q & \frac{1}{2} \partial_{x}-q \partial_{x}^{-1} q^{*} \\
\frac{1}{2} \partial_{x}-q^{*} \partial_{x}^{-1} q & q^{*} \partial_{x}^{-1} q^{*}
\end{array}\right),
\end{aligned}
$$

while the conserved densities become

$$
H^{1}=-\int\left(q_{x} q_{x}^{*}+q^{2} q^{* 2}\right) d x \quad \text { and } \quad H^{2}=i \int\left(q_{x}^{*} q-q_{x} q^{*}\right) d x .
$$

Under the parity-time (PT) preserving symmetry which generates the nonlocal nonlinear Schrödinger equation, we take

$$
r(x, t)=q^{*}(-x, t),
$$

whereby (3) gives the pair of nonlocal nonlinear Schrödinger equations (NNS), viz.,

$$
\begin{aligned}
q_{t}(x, t) & =-i q_{x x}(x, t)+2 i q^{2}(x, t) q^{*}(-x, t), \\
q^{*}(-x, t)_{t} & =i q_{x x}^{*}(-x, t)-2 i q^{* 2}(-x, t) q(x, t) .
\end{aligned}
$$

The foregoing equations are nonlocal since the evolution of the dynamical variable at the transverse coordinate $\mathrm{x}$ always requires information from the opposite point $(-x)$.

To check PT symmetry, the first of the equations in (12) is rewritten as

$$
i q_{t}(x, t)=q_{x x}(x, t)+V(q, x, t) q(x, t),
$$

where

$$
V(q, x, t)=-2 q(x, t)+q^{*}((-x, t)
$$

is the self-induced potential.

It is easy to show that

$$
V(q, x, t)=V^{*}(q,-x, t),
$$

which establishes the PT symmetry condition. 
The bi-Hamiltonian structure of the pair of $N N S$ equations are given by

$$
\begin{aligned}
B_{N N S}^{1} & =\left(\begin{array}{cc}
0 & -i \\
i & 0
\end{array}\right), \\
B_{N N S}^{2} & =\left(\begin{array}{cc}
q(x, t) \partial_{x}^{-1} q(x, t) & \frac{1}{2} \partial_{x}-q(x, t) \partial_{x}^{-1} q^{*}(-x, t) \\
\frac{1}{2} \partial_{x}-q^{*}(-x, t) \partial_{x}^{-1} q(x, t) & q^{*}(-x, t) \partial_{x}^{-1} q^{*}(-x, t)
\end{array}\right) .
\end{aligned}
$$

Here the arguments of $q$ and $q^{*}$ are written out explicitly to emphasize the nonlocal character of the equations.

The corresponding conserved densities are

$$
H_{N N S}^{1}=-\int\left(q_{x} q_{x}^{*}+q^{2} q^{* 2}\right) d x, \quad H_{N N S}^{2}=i \int\left(q_{x}^{*} q-q_{x} q^{*}\right) d x .
$$

As before, $q=q(x, t)$ and $q^{*}=q^{*}(-x, t)$ in the immediately preceding equation.

\section{Nonholonomic deformation of the nonlocal nonlinear Schrödinger equation}

\subsection{The bi-Hamiltonian approach}

In this formalism, one uses $(i)$ the bi-Hamiltonian structure of the parent equation and $(i i)$ deforming variables, to obtain the deformed equations as well as the constraint conditions on the deforming functions. In the present case, the pair of deformed nonlocal nonlinear Schrödinger equations may be expressed as

$$
\begin{aligned}
\left(\begin{array}{c}
q(x, t) \\
q^{*}(-x, t)
\end{array}\right)_{t} & =B_{N N S}^{1}\left(\begin{array}{c}
\frac{\delta}{\delta q} \\
\frac{\delta}{\delta q^{*}}
\end{array}\right) H_{N N S}^{1}-B_{N N S}^{1}\left(\begin{array}{c}
g(x, t) \\
g^{*}(-x, t)
\end{array}\right) \\
& =B_{N N S}^{2}\left(\begin{array}{c}
\frac{\delta}{\delta q} \\
\frac{\delta}{\delta q^{*}}
\end{array}\right) H_{N N S}^{2}-B_{N N S}^{1}\left(\begin{array}{c}
g(x, t) \\
g^{*}(-x, t)
\end{array}\right),
\end{aligned}
$$

where $g(x, t)$ and $g^{*}(-x, t)$ are the perturbing functions, $*$ denotes the complex conjugate, and the argument of the complex conjugate is taken suitably to fit into the $N N S$ scheme.

Following the method adopted in [32], (18) yields the following pair of deformed nonlocal nonlinear Schrödinger equations:

$$
\begin{aligned}
q_{t}(x, t) & =-i q_{x x}(x, t)+2 i q^{*}(-x, t) q^{2}(x, t)+i g^{*}(-x, t), \\
q_{t}^{*}(-x, t) & =i q_{x x}^{*}(-x, t)-2 i q^{* 2}(-x, t) q(x, t)-i g(x, t) .
\end{aligned}
$$

The constraints on the perturbing variables $g(x, t)$ and $g^{*}(-x, t)$ are obtained by setting

$$
B_{N N S}^{2}\left(\begin{array}{c}
g(x, t) \\
g^{*}(-x, t)
\end{array}\right)=0
$$


which leads to the conditions

$$
\begin{gathered}
g_{x}^{*}(-x, t)+2 q(x, t) \partial^{-1}\left[q(x, t) g(x, t)-q^{*}(-x, t) g^{*}(-x, t)\right]=0 \\
g_{x}(x, t)+2 q^{*}(-x, t) \partial^{-1}\left[q^{*}(-x, t) g^{*}(-x, t)-q(x, t) g(x, t)\right]=0 .
\end{gathered}
$$

Thus, the constraints, as they stand, are integro-differential in nature.

Multiplying (22) by $q^{*}(-x, t)$ and $(23)$ by $q(x, t)$ and adding, one obtains

$$
q(x, t) g_{x}(x, t)+q^{*}(-x, t) g_{x}^{*}(-x, t)=0 .
$$

\subsection{Lax pair approach}

To construct the NHD in this method, one starts with a Lax pair, keeping the space part $U(\lambda)$ unchanged, but modifying the temporal component $V(\lambda)$. This implies that the scattering problem remains unchanged, but the time evolution of the spectral data becomes different in the perturbed models. To retain integrability, the nonholonomic constraints have to be affine in velocities prohibiting explicit velocity dependence of the deformed dynamical system. This requires deformation of the temporal component of the Lax pair only as in the absence of its time derivative in the flatness condition the dynamical equation can remain velocityindependent [41]. It is due to such construction that the system can retain its integrability in spite of being subject to perturbation.

Under the $P T$ symmetric transformation, the Lax pair takes the form

$$
\begin{gathered}
U=-i \lambda \sigma_{3}+q(x, t) \sigma_{+} q^{*}(-x, t) \sigma_{-}, \\
V_{\text {original }}=\left[2 i \lambda^{2}+i q(x, t) q^{*}(-x, t)\right] \sigma_{3}-\left[2 \lambda q(x, t)+i q_{x}(x, t)\right] \sigma_{+}+ \\
+\left[-2 \lambda q^{*}(-x, t)+i q_{x}^{*}(-x, t)\right] \sigma_{-},
\end{gathered}
$$

where $V_{\text {original }}$ is the undeformed temporal component of the Lax pair and the arguments of the variables have been explicitly shown to emphasize the presence of nonlocality.

The only scale present in the system is the spectral parameter $\lambda$, defining the corresponding solution space. In order to obtain a deformation of the temporal part, which maintains integrability through the flatness condition of the type in Eq. (4), it is intuitively obvious that the deformation part will be a function of $\lambda$. We propose the following additive deformation term to the temporal Lax component of the nonlocal NLS equation:

$$
V_{\text {deformation }}=\frac{1}{2} \lambda^{-1}\left[a(x, t) \sigma_{3}+b(x, t) \sigma_{+}+c(x, t) \sigma_{-}\right] .
$$

The adopted deformation of Eq. (27) contains only $\mathcal{O}\left(\lambda^{-1}\right)$ terms. The higher-order terms in $\lambda$ (zero or positive powers) only lead to additional perturbed dynamical systems at each order, and vanish when the terms are substituted order-by-order. This is because the nonlocal NLS equations arise from contributions of $\mathcal{O}\left(\lambda^{0}\right)$ in the flatness condition, and the presence of any higher-order contribution is decoupled from the dynamics governed by the same. Therefore, the highest-order deformations end up yielding trivial identities, which eventually eliminates all the contributions with positive powers of $\lambda$ in the nonlocal NLS equation. One can verify this by adding a term with positive powers of $\lambda$ to $V_{\text {deformed }}$. Therefore, the expression in Eq. (27) is general as far as zero or positive powers of $\lambda$ are concerned. 
Taking $\tilde{V}=V_{\text {original }}+V_{\text {deformation }}$ and imposing the zero curvature or flatness condition, the nonholonomic deformed equations are obtained as the $\mathcal{O}\left(\lambda^{0}\right)$ terms in the above condition

$$
\begin{gathered}
q_{t}(x, t)+i q_{x x}(x, t)-2 i q^{2}(x, t) q^{*}(-x, t)-i b=0, \\
q_{t}^{*}(-x, t)-i q_{x x}^{*}(-x, t)+2 i q^{* 2}(-x, t) q(x, t)+i c=0 .
\end{gathered}
$$

Putting $b(x, t)=g^{*}(-x, t)$ and $c(x, t)=g(x, t)$ in Eqs. (28) and (29), respectively, we are led to the nonholonomic deformed coupled nonlocal NLS equations

$$
\begin{gathered}
q_{t}(x, t)=-i q_{x x}(x, t)+2 i q^{2}(x, t) q^{*}(-x, t)+i g^{*}(-x, t), \\
q_{t}^{*}(-x, t)=i q_{x x}^{*}(-x, t)-2 i q^{* 2}(-x, t) q(x, t)-i g(x, t) .
\end{gathered}
$$

Such deformations are trivially expected and they effectively make the original nonlocal NLS equations inhomogeneous by introducing source terms $g$ and $g^{*}$ into the dynamics. Such equations are already known to form integrable systems and thus satisfy our primary goal.

The differential constraints on the perturbing variables are obtained from the $\mathcal{O}\left(\lambda^{-1}\right)$ sector in the flatness condition, on equating the coefficients of the generators $\sigma_{3}, \sigma_{+}$and $\sigma_{-}$:

$$
\begin{gathered}
a_{x}=q(x, t) g(x, t)-q^{*}(-x, t) g^{*}(-x, t), \\
g_{x}^{*}(-x, t)+2 q(x, t) a(x, t)=0, \\
g_{x}(x, t)-2 q^{*}(-x, t) a(x, t)=0 .
\end{gathered}
$$

Eliminating $a(x, t)$ from the above equations, we obtain a single differential constraint as

$$
\begin{aligned}
q(x, t) g_{x x}(x, t)+q_{x}^{*}(-x, t) g_{x}^{*}( & -x, t)+ \\
+ & 2 q(x, t) q^{*}(-x, t)\left[q^{*}(-x, t) g^{*}(-x, t)-q(x, t) g(x, t)\right]=0 .
\end{aligned}
$$

Using Eqs. (30) and (31) to eliminate the remaining perturbing variables $g(x, t)$ and $g^{*}(-x, t)$ from Eq. (35), we derive a single higher-order nonholonomic deformed nonlocal nonlinear Schrödinger equation given as

$$
\begin{aligned}
& q(x, t)\left[\partial^{2}-2 q(x, t) q^{*}(-x, t)\right]\left[i q_{t}^{*}(-x, t)+q_{x x}^{*}(-x, t)-2 q(x, t) q^{* 2}(-x, t)\right] \\
& +q_{x}^{*}(-x, t)\left[-i q_{t}(x, t)+q_{x x}(x, t)-2 q^{2}(x, t) q^{*}(-x, t)\right]_{x} \\
& +2 q(x, t) q^{* 2}(-x, t)\left[-i q_{t}(x, t)+q_{x x}(x, t)-2 q^{2}(x, t) q^{* 2}(-x, t)\right] .
\end{aligned}
$$

Such an equation is subjected to the dynamics of Eqs. (30) and (31), and therefore does not yield any new dynamics, and eventually reflects the constraint itself in a different form. This is in accordance with the previous argument that no term, with power of $\lambda$ other than that responsible for yielding Eqs. (30) and (31), can yield dynamics to the nonlocal NLS system, as it will violate the overall integrability of the system itself.

The constraint of Eq. (35) is nonholonomic in nature, as it contains differentials of corresponding variables, and characterizes the corresponding deformation. It is crucial that such a constraint solely arises from the terms with negative power of the spectral parameter. Further, explicit forms of the local functions $a, g_{1}$ and $g_{2}$ are not necessary to establish the integrability, and they represent a class that satisfies the constraint in Eq. (35). In other words, the constraints arise form $\mathcal{O}\left(\lambda^{-1}\right)$ contributions, and additionally restrict the allowed values of $q(x, t)$ and $q^{*}(-x,-t)$ of the deformed dynamics of $\mathcal{O}\left(\lambda^{0}\right)$. 
From (33) and (34) we obtain

$$
q^{*}(-x, t) g_{x}^{*}(-x, t)+q(x, t) g_{x}(x, t)=0,
$$

which agrees with (24), thereby establishing the equivalence of the bi-Hamiltonian and Lax pair approaches.

\section{Derivative nonlinear Schrödinger equations and their bi-Hamiltonian structure}

\subsection{The coupled Kaup-Newell system}

We consider the Lax pair of the Kaup - Newell (KN) system which belongs to the derivative nonlinear Schrödinger family of integrable systems. This is given by

$$
\begin{gathered}
U=-i \lambda^{2} \sigma_{3}+\lambda q \sigma_{+}+\lambda r \sigma_{-}, \\
V=-i\left(2 \lambda^{4}+\lambda^{2} q r\right) \sigma_{3}+\left[2 \lambda^{3} q+\lambda\left(i q_{x}+q^{2} r\right)\right] \sigma_{+}+\left[2 \lambda^{3} r+\lambda\left(-i r_{x}+q r^{2}\right)\right] \sigma_{-} .
\end{gathered}
$$

The zero curvature condition leads to the following coupled Kaup-Newell system of equations:

$$
\begin{aligned}
& q_{t}(x, t)=i q_{x x}(x, t)+\left(q^{2}(x, t) r(x, t)\right)_{x}, \\
& r_{t}(x, t)=-i r_{x x}(x, t)+\left(q(x, t) r^{2}(x, t)\right)_{x} .
\end{aligned}
$$

The bi-Hamiltonian structure of the Kaup - Newell system is given by

$$
\begin{aligned}
& B_{K N}^{1}=\left(\begin{array}{ll}
0 & \partial \\
\partial & 0
\end{array}\right), \\
& B_{K N}^{2}=B_{K N}^{1} J_{K N} B_{K N}^{1},
\end{aligned}
$$

where

$$
J=\left(\begin{array}{cc}
r \partial^{-1} r & i+r \partial^{-1} q \\
i+q \partial^{-1} r & q \partial^{-1} q
\end{array}\right)
$$

The conserved densities are

$$
H_{K N}^{1}=\frac{1}{2} \int\left(q^{2} r^{2}+i\left(q_{x} r-q r_{x}\right)\right) d x \quad \text { and } \quad H^{2}=\int(q r) d x .
$$

\subsection{Symmetry reduction and the nonlocal coupled Kaup-Newell system}

We impose the symmetry reduction

$$
r(x, t)=q(-x,-t) .
$$

Under this reduction the system of equations (40) and (41) is compatible and we obtain the reverse space-time nonlocal coupled "classical" DNLS equations or the nonlocal coupled KaupNewell system given below:

$$
\begin{aligned}
q_{t}(x, t) & =i q_{x x}(x, t)+\left(q^{2}(x, t) q(-x,-t)\right)_{x}, \\
q_{t}(-x,-t) & =-i q_{x x}(-x,-t)+\left(q(x, t) q^{2}(-x,-t)\right)_{x} .
\end{aligned}
$$


The nonlocal character of the equations arises from the fact that the evolution of the field at given spatial and temporal coordinates $x$ and $t$ is governed not only by the values of the field at these coordinates, but also by the information coming from the corresponding reversed coordinates, viz., $(-x)$ and $(-t)$.

The bi-Hamiltonian structure of the above equations is given by

$$
\begin{aligned}
& B_{N K N}^{1}=\left(\begin{array}{ll}
0 & \partial \\
\partial & 0
\end{array}\right), \\
& B_{N K N}^{2}=B_{N K N}^{1} J_{N K N} B_{N K N}^{1},
\end{aligned}
$$

where

$$
J_{N K N}=\left(\begin{array}{cc}
q(-x,-t) \partial^{-1} q(-x,-t) & i+q(-x,-t) \partial^{-1} q(x, t) \\
i+q(x, t) \partial^{-1} q(-x,-t) & q(x, t) \partial^{-1} q(x, t)
\end{array}\right) .
$$

Here $N K N$ denotes the nonlocal Kaup-Newell system.

The conserved densities are given by

$$
\begin{aligned}
& H_{N K N}^{1}=\frac{1}{2} \int\left(q^{2}(x, t) q^{2}(-x,-t)+i\left(q_{x}(x, t) q(-x,-t)-q(x, t) q_{x}(-x,-t)\right)\right) d x, \\
& H_{N K N}^{2}=\int(q(x, t) q(-x,-t)) d x .
\end{aligned}
$$

\section{Nonholonomic deformation of the nonlocal coupled Kaup - Newell system}

The methods and arguments adopted while deriving the nonholonomic deformation of the nonlocal coupled Kaup-Newell system are similar to those followed in the case of the nonlocal coupled nonlinear Schrödinger equations.

\subsection{The bi-Hamiltonian approach}

In this formalism, the nonlocal coupled Kaup - Newell systems, under nonholonomic deformation, are expressed in terms of the bi-Hamiltonian structure and the conserved densities as

$$
\begin{aligned}
\left(\begin{array}{c}
q(x, t) \\
q(-x,-t)
\end{array}\right)_{t} & =B_{N K N}^{1}\left(\begin{array}{c}
\frac{\delta}{\delta q(x, t)} \\
\frac{\delta}{\delta q(-x,-t)}
\end{array}\right) H_{N K N}^{1}-B_{N K N}^{1}\left(\begin{array}{c}
g^{1}(x, t) \\
g^{2}(-x,-t)
\end{array}\right) \\
& =B_{N K N}^{2}\left(\begin{array}{c}
\frac{\delta}{\delta q(x, t)} \\
\frac{\delta}{\delta q(-x,-t)}
\end{array}\right) H_{N K N}^{2}-B_{N K N}^{1}\left(\begin{array}{c}
g^{1}(x, t) \\
g^{2}(-x,-t)
\end{array}\right) .
\end{aligned}
$$

Here $g^{1}(x, t)$ and $g^{2}(-x,-t)$ are the perturbing functions, and the argument of the second function is suitably modified to fit the reverse space-time nonlocal character of the parent equations.

From Eq. (51), the pair of deformed nonlocal coupled $K N$ equations are

$$
\begin{aligned}
q_{t}(x, t) & =i q_{x x}(x, t)+\left(q^{2}(x, t) q(-x,-t)\right)_{x}-g_{x}^{2}(-x,-t), \\
q_{t}(-x,-t) & =-i q_{x x}(-x,-t)+\left(q^{2}(-x,-t) q(x, t)\right)_{x}-g_{x}^{1}(x, t) .
\end{aligned}
$$


The constraints on the perturbing variables $g^{1}(x, t)$ and $g^{2}(-x,-t)$ are obtained by setting

$$
B_{N K N}^{2}\left(\begin{array}{c}
g^{1}(x, t) \\
g^{2}(-x,-t)
\end{array}\right)=0,
$$

and yield the following equations:

$$
\begin{gathered}
i g_{x x}^{2}(-x,-t)+(q(x, t) B)_{x}=0, \\
-i g_{x x}^{1}(x, t)+(q(-x,-t) B)_{x}=0,
\end{gathered}
$$

where $B=\partial^{-1} A$, and

$$
A=q(x, t) g_{x}^{1}(x, t)+q(-x,-t) g_{x}^{2}(-x,-t)=0 .
$$

\subsection{Lax pair approach}

To derive the nonholonomic deformation in the case of the nonlocal Kaup-Newell system, one starts with the following Lax pair:

$$
\begin{gathered}
U=-i \lambda^{2} \sigma_{3}+\lambda q(x, t) \sigma_{+}+\lambda q(-x,-t) \sigma_{-}, \\
V_{\text {original }}=\left(-2 i \lambda^{4}-i \lambda^{2} q(x, t) q(-x,-t)\right) \sigma_{3} \\
+\left(2 \lambda^{3} q(x, t)+\lambda\left(i q_{x}(x, t)+q^{2}(x, t) q(-x,-t)\right)\right) \sigma_{+} \\
+\left(2 \lambda^{3} q(-x,-t)+\lambda\left(-i q_{x}(-x,-t)+q(x, t) q^{2}(-x,-t)\right)\right) \sigma_{-},
\end{gathered}
$$

where $V_{\text {original }}$ denotes the undeformed temporal component of the Lax pair generating the nonlocal coupled Kaup - Newell system of equations.

We introduce deformation in the time component above by defining

$$
V_{\text {deformed }}=\alpha\left(G^{0}+\lambda^{-1} G^{1}+\lambda^{-2} G^{2}\right),
$$

where

$$
\begin{aligned}
& G^{(0)}=w \sigma_{3}+m_{1} \sigma_{+}+m_{2} \sigma_{-}, \\
& G^{(1)}=a \sigma_{3}+g_{1} \sigma_{+}+g_{2} \sigma_{-}, \\
& G^{(2)}=b \sigma_{3}+f_{1} \sigma_{+}+f_{2} \sigma_{-} .
\end{aligned}
$$

Taking $\tilde{V}=V_{\text {original }}+V_{\text {deformed }}$ and using the zero curvature condition on $U$ defined by (58) and $\tilde{V}$, we arrive at the following results for the variables contained in the functions $G^{0}, G^{1}$ and $G^{2}: m_{1}=0, m_{2}=0, a=0, f_{1}=0, f_{2}=0$ and $b=b(t)$, where the last entry indicates that $b$ is a function of $t$ only.

The pair of nonholonomic deformed coupled nonlocal Kaup-Newell equations are given below:

$$
\begin{gathered}
q_{t}(x, t)-i q_{x x}(x, t)-\left(q^{2}(x, t) q(-x,-t)\right)_{x}-2 i \alpha g_{1}-2 \alpha w q(x, t)=0, \\
q_{t}(-x,-t)+i q_{x x}(-x,-t)-\left(q^{2}(-x,-t) q(x, t)\right)_{x}+2 i \alpha g_{2}-2 \alpha w q(-x,-t)=0 .
\end{gathered}
$$

The differential constraints on the nonzero variables $w, g_{1}$ and $g_{2}$ are given by

$$
\begin{gathered}
w_{x}=q(x, t) g_{2}-q(-x,-t) g_{1}, \\
g_{1 x}+2 b(t) q(x, t)=0, \\
g_{2 x}-2 b(t) q(-x,-t)=0 .
\end{gathered}
$$


We now try to obtain new nonlinear integrable systems by resolving the constraint relations and expressing the perturbing variables in terms of the basic field variables. To this end, let us put

$$
\begin{aligned}
& q(x, t)=u_{x}(x, t), \\
& q(-x,-t)=v_{x}(-x,-t) .
\end{aligned}
$$

Using (69) in (66),(67) and (68) we are led to the following relations:

$$
\begin{aligned}
& g_{1}=-2 b(t) u(x, t), \\
& g_{2}=2 b(t) v(-x,-t), \\
& w=2 b(t) u(x, t) v(-x,-t)+K(t) .
\end{aligned}
$$

Eliminating $g_{1}, g_{2}$ and $w$ from (64) and (65) by using (70), we can rewrite the nonholonomic deformed coupled nonlocal (space and time reversed) Kaup-Newell system as

$$
\begin{gathered}
u_{x t}(x, t)-i u_{x x x}(x, t)-\left(u_{x}^{2}(x, t) v_{x}(-x,-t)\right)_{x}+4 i \alpha b(t) u(x, t) \\
-2 \alpha u_{x}(x, t)(2 b(t) u(x, t) v(-x,-t)+K(t))=0, \\
v_{x t}(-x,-t)+i v_{x x x}(-x,-t)-\left(u_{x}(x, t) v_{x}^{2}(-x,-t)\right)_{x}+4 i \alpha b(t) v(-x,-t) \\
+2 \alpha v_{x}(-x,-t)(2 b(t) u(x, t) v(-x,-t)+K(t))=0 .
\end{gathered}
$$

Equations (71) and (72) are coupled nonlocal evolution equations that are nonautonomous in character with arbitrary time-dependent coefficients $b(t)$ and $K(t)$. The constraints have been resolved and thus removed from the system. These equations may be regarded as generalized nonlocal versions of the Lennels - Fokas equations with the inclusion of a nonlinear derivative term as well as a higher-order dispersion term.

\section{Discussion and conclusion}

The study of nonlocal integrable systems is a relatively new area in the domain of integrable systems. The results in this paper are significant in two different ways. Firstly, from a mathematical point of view, we have presented integrable nonlocal equations which have some physical applications in PT-symmetric quantum physics. The bi-Hamiltonian structure of these nonlocal equations has been clearly furnished as part of the analysis. Secondly, this is probably the first work in which such systems have been studied under the lens of nonholonomic deformation, which is a perturbing method that retains integrability of the system. The deformation is carried out using the bi-Hamiltonian formalism due to Kupershmidt, as well as the Lax pair approach. The systems chosen were those belonging to the nonlinear Schrödinger family, since the nonlinear Schrödinger equation is one of the most celebrated equations having a number of physical applications.

Our next program will be to study the soliton and multisoliton solutions of these new sets of nonlocal NLS equations, particularly, whether their solutions give rise to accelerating solitons or some such interesting phenomena under a suitable choice of the explicitly time-dependent coefficients. It will also be interesting to study other aspects of these systems, such as their behavior under quasi-integrable deformation (QID) [42, 43], obtaining them under reduction of the self-dual Yang-Mills (SDYM) equations [44, 45] and their connection with other integrable systems [46]. These topics will form the basis of our future investigations in this area [47]. 


\section{Acknowledgments}

We would like to express our sincere appreciation to Professor Sarbarish Chakravarty for his encouragement and enlightening discussions. We would also like to thank Dr. Kumar Abhinav for his interest.

\section{An observation}

A large number of papers have been published on nonlocal integrable systems in the past few years. We have cited only those papers which have a direct bearing on and relevance to the present work.

\section{References}

[1] Das, A., Integrable Models, World Sci. Lecture Notes Phys., vol. 30, Teaneck, N.J.: World Sci., 1989.

[2] Lax, P. D., Integrals of Nonlinear Equations of Evolution and Solitary Waves, Comm. Pure Appl. Math., 1968, vol.21, pp. 467-490.

[3] Ablowitz, M. J. and Clarkson, P. A., Solitons, Nonlinear Evolution Equations and Inverse Scattering, London Math. Soc. Lecture Note Ser., vol. 149, Cambridge: Cambridge Univ. Press, 1991.

[4] Ablowitz, M. J., Kaup, D. J., Newell, A. C., and Segur, H., The Inverse Scattering Transform: Fourier Analysis for Nonlinear Problems, Stud. Appl. Math., 1974, vol. 53, no. 4, pp. 249-315.

[5] Faddeev, L.D. and Takhtajan, L.A., Hamiltonian Methods in the Theory of Solitons, Berlin: Springer, 1987.

[6] Magri, F., A Simple Model of the Integrable Hamiltonian Equation, J. Math. Phys., 1978, vol.19, no. 5 , pp. $1156-1162$.

[7] Malomed, B., Nonlinear Schrödinger Equations, in Encyclopedia of Nonlinear Science, A. Scott (Ed.), New York: Routledge, 2005, pp. 639-643.

[8] Zhidkov, P.E., Korteweg-de Vries and Nonlinear Schrödinger Equations: Qualitative Theory, Lecture Notes in Math., vol.1756, Berlin: Springer, 2001.

[9] Rogers, C. and Schief, W., Bäcklund and Darboux Transformations: Geometry and Modern Applications in Soliton Theory, Cambridge Texts Appl. Math., vol. 30, Cambridge: Cambridge Univ. Press, 2002.

[10] Ablowitz, M. and Musslimani, Z., Integrable Nonlocal Nonlinear Schrödinger Equation, Phys. Rev. Lett., 2013, vol.110, no. 6, 064105, 5 pp.

[11] Guo, A., Salamo, G.J., Duchesne, D., Morandotti, R., Volatier-Ravat, M., Aimez, V., Siviloglou, G. A., and Christodoulides, D. N., Observation of PT-Symmetry Breaking in Complex Optical Potentials, Phys. Rev. Lett., 2009, vol. 103, no. 9, 093902, 4 pp.

[12] Regensburger, A., Bersch, C., Miri, M. A., Onishchukov, G., Christodoulides, D. N., and Peschel, U., Parity-Time Synthetic Photonic Lattices, Nature, 2012, vol. 488, no. 7410, pp. 167-171.

[13] Rüter, Ch.E., Makris, K. G., El-Ganainy, R., Christodoulides, D. N., Segev, M., and Kip, D., Observation of Parity-Time Symmetry in Optics, Nature Phys., 2010, vol. 6, no. 3, pp. 192-195.

[14] Fokas, A.S., Its, A.R., and Sung, L.-Y., The Nonlinear Schrödinger Equation on the Half-Line, Nonlinearity, 2005, vol. 18, no. 4, pp. 1771-1822.

[15] Fokas, A. S., Integrable Nonlinear Evolution Equations on the Half-Line, Comm. Math. Phys., 2002, vol. 230, no. 1, pp. 1-39.

[16] Fokas, A. S. and Its, A.R., The Nonlinear Schrödinger Equation on the Interval, J. Phys. A, 2004, vol. 37, no. 23, pp.6091-6114. 
[17] Fokas, A. S. and Its, A. R., The Linearization of the Initial-Boundary Value Problem of the Nonlinear Schrödinger Equation, SIAM J. Math. Anal., 1996, vol. 27, no. 3, pp. 738-764.

[18] Its, A. R., Asymptotic Behavior of the Solutions to the Nonlinear Schrödinger Equation, and Isomonodromic Deformations of Systems of Linear Differential Equations, Soviet Math. Dokl., 1981, vol. 24, no. 3, pp.452-456; see also: Dokl. Akad. Nauk SSSR, 1981, vol.261, no.1, pp. 14-18.

[19] Ablowitz, M.J., Fokas, A.S., and Musslimani, Z.H., On a New Nonlocal Formulation of Water Waves, J. Fluid Mech., 2006, vol. 562, pp. 313-344.

[20] Fokas, A. S., Integrable Multidimensional Versions of the Nonlocal Nonlinear Schrödinger Equation, Nonlinearity, 2016, vol. 29, no. 2, pp.319-324.

[21] Ablowitz, M. J. and Musslimani, Z. H., Integrable Nonlocal Nonlinear Equations, Stud. Appl. Math., 2017, vol. 139, no. 1, pp. 7-59.

[22] Ablowitz, M. J. and Musslimani, Z.H., Integrable Discrete PT Symmetric Model, Phys. Rev. E, 2014, vol. 90, no. 3, 032912, 5 pp.

[23] Ablowitz, M. J. and Musslimani, Z.H., Inverse Scattering Transform for the Integrable Nonlocal Nonlinear Schrödinger Equation, Nonlinearity, 2016, vol. 29, no. 3, pp. 915-946.

[24] Ablowitz, M. J., Luo, X.-D., and Musslimani, Z. H., Inverse Scattering Transform for the Nonlocal Nonlinear Schrödinger Equation with Nonzero Boundary Conditions, J. Math. Phys., 2018, vol. 59, no. 1, 011501, 42 pp.

[25] Valchev, T., On a Nonlocal Nonlinear Schrödinger Equation, in Mathematics in Industry, A. Slavova (Ed.), Cambridge: Cambridge Scholars Publ., 2014, pp. 36-52.

[26] Gürses, M. and Pekcan, A., Nonlocal Nonlinear Schrödinger Equations and Their Soliton Solutions, J. Math. Phys., 2018, vol.59, no. 5, 051501, 17 pp.

[27] Gerdjikov, V.S. and Saxena, A., Complete Integrability of Nonlocal Nonlinear Schrödinger Equation, J. Math. Phys., 2017, vol. 58, no. 1, 013502, 33 pp.

[28] Bender, C. M., PT Symmetry in Quantum and Classical Physics, Hackensack, N.J.: World Sci., 2019.

[29] Bender, C. M., Introduction to PT-Symmetric Quantum Theory, arXiv:quant-ph/0501052 (2005).

[30] Bender, C. M., PT Symmetric Quantum Theory, J. Phys. Conf. Ser., 2015, vol. 631, 012002, 12 pp.

[31] Karasu-Kalkanli, A., Karasu, A., Sakovich, A., Sakovich, S., and Turhan, R., A New Integrable Generalization of the Korteweg-de Vries Equation, J. Math. Phys., 2008, vol.49, no. 7, 073516, $10 \mathrm{pp}$.

[32] Kuperschmidt, B. A., KdV6: An Integrable System, Phys. Lett. A, 2008, vol. 372, no. 15, pp. 26342639.

[33] Kundu, A., Exact Accelerating Solitons in Nonholonomic Deformation of the KdV Equation with Two-Fold Integrable Hierarchy, J. Phys. A, 2008, vol.41, no. 49, 495201, 7 pp.

[34] Kundu, A., Sahadevan, R., and Nalinidevi, L., Nonholonomic Deformation of KdV and mKdV Equations and Their Symmetries, Hierarchies and Integrability, J. Phys. A, 2009, vol.42, no. 11, 115213, 13 pp.

[35] Kundu, A., Two-Fold Integrable Hierarchy of Nonholonomic Deformation of the Derivative Nonlinear Schrödinger and the Lenells-Fokas Equation, J. Math. Phys., 2010, vol. 51, no. 2, 022901, 17 pp.

[36] Guha, P., Nonholonomic Deformation of Generalized KdV-Type Equations, J. Phys. A, 2009, vol. 42, no. 34, 345201, 17 pp.

[37] Guha, P., Nonholonomic Deformation of Coupled and Supersymmetric KdV Equations and EulerPoincaré-Suslov Method, Rev. Math. Phys., 2015, vol. 27, no. 4, ID 1550011-25.

[38] Abhinav, K., Guha, P., and Mukherjee, I., Study of Quasi-Integrable and Non-Holonomic Deformation of Equations in the NLS and DNLS Hierarchy, J. Math. Phys., 2018, vol.59, no. 10, 101507, $18 \mathrm{pp}$.

[39] Fokas, A. S. and Pelloni, B., A Transform Method for Evolution PDEs on the Interval, IMA J. Appll. Math., 2005, vol. 70, no. 4, pp. 564-587. 
[40] Fokas, A. S. and Pelloni, B., Unified Transform for Boundary Value Problems: Applications and Advances, Philadelphia, Pa.: SIAM, 2014.

[41] Krupková, O., Mechanical Systems with Nonholonomic Constraints, J. Math. Phys., 1997, vol.38, no. 10, pp. 5098-5126.

[42] Ferreira, L. A. and Zakrzewski, W. J., The Concept of Quasi-Integrability: A Concrete Example, J. High Energy Phys., 2011, no. 5, 130, 39 pp.

[43] Blas, H. and Zambrano, M., Quasi-Integrability in the Modified Defocusing Non-Linear Schrödinger Model and Dark Solitons, J. High Energy Phys., 2016, no. 3, 005, front matter +47 pp.

[44] Ablowitz, M. J., Chakravarty, S., and Takhtajan, L. A., A Self-Dual Yang-Mills Hierarchy and Its Reductions to Integrable Systems in $1+1$ and 2+1 Dimensions, Comm. Math. Phys., 1993, vol. 158, no. 2, pp. 289-314.

[45] Ablowitz, M. J., Chakravarty, S., and Halburd, R. G., Integrable Systems and Reductions of the SelfDual Yang-Mills Equations: Integrability, Topological Solitons and Beyond, J. Math. Phys., 2003, vol. 44, no. 8, pp. 3147-3173.

[46] Ablowitz, M.J. and Musslimani, Z.H., Integrable Nonlocal Asymptotic Reductions of Physically Significant Nonlinear Equations, arXiv:1903.06752 (2019).

[47] Mukherjee, I., Guha, P., and Abhinav, K., Studies on Different Aspects of Nonlocal Integrable Systems: Soliton Solutions, Deformations and Reductions from SDYM Equations, in preparation (2019). 